\title{
Awareness Solutions for Informal Communication Negotiation Support at Work
}

\author{
Agnieszka Matysiak Szóstek \\ Industrial Design, Eindhoven University of Technology, Den Dolech 2, \\ 5600MB Eindhoven, The Netherlands \\ a.matysiak@tue.nl
}

\begin{abstract}
The goal of our project is to design and evaluate an awareness system that supports handling interruptions for both interruption actors: interruptees and interruptors.
\end{abstract}

\section{Introduction}

Informal communication between coworkers is considered as an optimal way to exchange rapid feedback, share local context and reference common depictions [2]. But it comes at a cost: interruptions. If interruptions occur at wrong moments they cause loss of concentration, anxiety and time pressure [1]. However, once initiated at right times they serve as natural breaks and provide content for expressive interaction between colleagues. Current solutions supporting handling interruptions assume the interruptee's availability to be the best predictor of an appropriate interruption moment [2]. Yet, interruptors seem not to fully adhere to those availability indications as the proposed systems fail to help them convey their communicative needs. Also interruptees seem to experience social responsibility and they often reevaluate their availability according to the actual needs of their interruptors [4].

\section{Information Needs in Negotiating Interruptions}

A key challenge in designing awareness systems supporting handling interruptions lies in provision of means for expressing information needs that reach beyond simple availability indications. Participants in our studies reported needs that would help them to increase efficiency in interruption negotiation, which can be grouped in three groups: those related to social proximity between the actors, those defining the nature of the interruption subject and those determining the anticipated interruption duration. In order to determine whether our classification is accurate we have constructed two Technology Probes out of two interconnected devices: one standing in one's office and another located in front of one's door. At any time of the day the office occupant could indicate his/her communicative status on the office probe and the interruptor his/her information needs on the corridor probe. The evaluation of the probes showed that despite the probe straightforwardness and the simplicity of the proposed interaction a possibility of sharing relevant information needs was highly valued by both interruptors and interruptees. 


\section{Social and System Influence on Interruption Behaviour}

As the next step we set out to verify in what way different system and social behaviours influence interruptions. We have conducted an experiment testing the system condition, which offered a manual or an automatic approach to handling interruptions and the social condition, which identified two relationships: the Team representing people sharing a common goal and the Group representing those who did not share a goal but assumed social reciprocation. The results show that actors who share a common goal behave in a more sensitive manner when handling interruptions than those who do not share it. The choice between the manual and automatic system behaviour seems to depend on moment-to-moment activities of both actors rather than their social relationship. Participants in both roles and in both social conditions liked the possibility to select system behaviour to fit their current task.

\section{Future Work and Conclusions}

Through this research we aim to i) extend the current knowledge about information needs essential for successful interruption negotiation ii) contribute an awareness system supporting negotiating interruptions that in its expressiveness reaches beyond availability indications and iii) produce guidelines informing the design of a future systems supporting informal communication at work.

The following step in our study is to design and evaluate an awareness system that addresses previously collected information needs and interruption handling strategies. In our design we want to consider two situations, in which the intent for initiating interruption is produced: a moment when the interruptor forms a plan to go and interrupt, while still remaining at his/her own workplace and a moment when the interruptor takes an opportunistic decision to interrupt when either passing by the interruptee's office or remaining at the same office that the interruptee is present in. In both cases the interruptor needs to be provided with the interruptee's status indication and must have an opportunity to present some of the interruption characteristics.

\section{References}

[1] Adamczyk, P.D., Bailey, B.P.: If not now, when?: the effects of interruption at different moments within task execution. In: CHI, ACM Press, New York (2004)

[2] Fogarty, J., Hudson, S., Atkeson, C., Avrahami, D., Forlizzi, J., Kiesler, S., Lee, J., Yang, J.: Predicting human interruptability with sensors. ACM Transactions (2005)

[3] Nardi, B., Whittaker, S.: The place of face-to-face communication in distributed work, Distributed Work. MIT Press, Cambridge (2001)

[4] Wiberg, M., Whittaker, S.: Managing availability: supporting lightweight negotiations to handle interruptions. ACM Transactions 1 (2005) 